\title{
Zebrafish as a Model for Cardiac Development and Diseases
}

\author{
Man Qi ${ }^{1,2,3}$ and Yi-Han Chen ${ }^{1,2,3,4^{*}}$ \\ ${ }^{1}$ Key Laboratory of Basic Research in Cardiology of the Ministry of Education of China, East Hospital, Tongji University School of Medicine, Shanghai 200120, China \\ ${ }^{2}$ Research Center for Translational Medicine, Tongji University School of Medicine, Shanghai 200120, China \\ ${ }^{3}$ Department of Cardiology, East Hospital, Tongji University School of Medicine, Shanghai 200120, China \\ ${ }^{4}$ Institute of Medical Genetics, Tongji University, Shanghai 200092, China
}

"Corresponding author: Yi-Han Chen, Key Laboratory of Basic Research in Cardiology of the Ministry of Education of China, East Hospital, Tongji University School of Medicine, Shanghai 200120, China, Tel: +086-21-65989086; Fax: +086-21-65989086; E-mail: yihanchen@tongji.edu.cn

Rec Date: November 10, 2014 Acc Date: November 12, 2014 Pub Date: November 15, 2014

Copyright: ( 2015 Liu M, et al. This is an open-access article distributed under the terms of the Creative Commons Attribution License, which permits unrestricted use, distribution and reproduction in any medium, provided the original author and source are credited.

\section{Editorial}

The zebrafish has been widely used for studying organ development and diseases. This model has several excellent advantages, as follows: 1) an almost completely sequenced genome that is highly conserved with humans [1]; 2) in vitro fertilization capability, transparency of the embryos, easy to perform embryo operations and genetic manipulation $[2,3]$; 3) rapid growth, a short sexual maturation cycle, high fecundity; 4) well-established fluorescent protein-tracing strains and mutant strains; 5) heart regeneration ability after injury [4].

Zhang et al. knew these advantages very well and made very clever use of them. Their recent paper reported that they visually monitored the dynamic cellular events occurring in cardiac regeneration after injury of the zebrafish ventricle. These researchers also confirmed that a variety of cardiac lineages contribute to plasticity during myocardial injury, more than previously thought able to transdifferentiate to a new cell type. More importantly, their study identified potential cellular sources and strategies for generating new ventricular myocardium [5]. The methodology they adopted was to construct an ablation system (genetic ablation system) in the zebrafish [6] in which they began to ablate the ventricles at 3-4 days post-fertilization (dpf), an age when the heart has completed cardiac looping and cardiomyocytes in the cardiac chambers have fully differentiated [7] but the zebrafish remains optically clear. The targeted destruction of ventricular cardiomyocytes was induced by treatment with metronidazole and fluorescent proteins were used to separately track atrial (GFP) and ventricular (mCherry) myocardial cells during ventricular damage. With a combination of genetic fate mapping techniques [8], these authors revealed that differentiated atrial cardiomyocytes can transdifferentiate into ventricular cardiomyocytes to contribute to zebrafish cardiac ventricular regeneration. This transdifferentiation allowed atrial cardiomyocytes to divide and repair the damaged ventricle, a process that was closely related to the Notch signaling pathway, as transdifferentiation was blocked when Notch signaling was inhibited. The scientists plan to study the Notch signaling pathway as a next step, which will help to explore additional potential mechanisms in heart regeneration.

In addition to cell differentiation, heart development also involves cell specification as well as elaborate tissue remodeling [9]. The zebrafish offers unique advantages for exploring the genetic and molecular mechanisms of cardiac development and function because zebrafish embryos are amenable to Whole Mount In-situ Hybridization (WISH), especially when compared to mouse embryos, which makes it possible to directly observe how the heart develops and functions under stereomicroscopy, as opposed to sectioning, particularly in the early embryonic period. Another aspect is that a large number of fluorescent protein-labeled tissue-specific zebrafish strains have been well established, acting as a perfect genetic tracing system. Recent discoveries on early cardiac specification and the identification of the second heart field (SHF) in zebrafish take full advantage of this point. Zhou et al. performed many WISH experiments and generated different fluorescent strains to dynamically trace Ltbp3 in SHF formation in zebrafish. This research implicated Ltbp3, a regulator of Tgf secretion and activation, in arterial pole development in zebrafish. Knock-down of ltbp3 caused severe ventricular defects, with a lack of the cardiac structures derived from ltbp3+ cells due to compromised progenitor proliferation [10].

Heart development not only includes mechanical structure but also electrical activity, and the zebrafish has its own advantages in this regard. Isolated embryonic hearts are convenient for optical mapping and maintain biological activity for a very long time in medium, as opposed to the continuous perfusion required when performing a mouse or rat heart experiment. Daniela et al. identified a gradient of electrical coupling across the developing ventricular myocardium using high-speed optical mapping of transmembrane potentials and calcium concentrations in isolated zebrafish embryonic hearts. These authors demonstrated that non-canonical Wnt11 signals are required for the genesis of this myocardial electrical gradient and that Wnt11mediated attenuation of LTCC conductance is required in this process [11].

The final outcome of various heart diseases is decreased myocardial contractile function, which eventually develops into heart failure, and the zebrafish is also a very powerful tool in cardiac function research. One of our recently published articles on cardiac hypertrophy well presented this point. In our study, we found that tom70 acts as a molecular switch to determine pathological cardiac hypertrophy. To provide strong evidence of this, we employed the morpholino knockdown of tom70 in zebrafish and measured several cardiac functional parameters, including fractional shortening (FS), under high-speed imaging confocal microscopy using a clmc2-gfp-positive zebrafish strain. This experiment is as easy to perform as echocardiography on a mouse. We also performed whole-mount immunostaining to label the cell membrane and cell nucleus of the cardiomyocytes in zebrafish hearts and obtained three-dimensional images of the whole heart in $2 \mathrm{dpf}$ embryos to visually compare changes in cell size and cell number [12].

The zebrafish is currently widely applied in cardiovascular diseases to investigate the role of specific genes in cardiovascular development and disease occurrence through gene manipulation, and it can also act 
Citation: Qi M, Chen YH (2015) Zebrafish as a Model for Cardiac Development and Diseases. Human Genet Embryol 5: e112. doi:

Page 2 of 2

as a platform for large-scale screening of active cardiovascular substances, especially some new drugs. As an ideal model in drug screening, the zebrafish holds the advantages of high fecundity, conserved similarity to mammals, and ease with which large, phenotype-based screens can be performed. The organism not only simulates the physiological complexity of higher animals but also is an alternative to some mammalian models with a high cost-effective index, especially dogs and pigs, contributing to several fields of drug discovery, such as target identification, disease modeling, lead discovery and toxicology [13]. David et al. established a zebrafish model of cardiac repolarization by using fluorescent reporters of transmembrane potential and then conducted a drug-sensitized genetic screen in zebrafish; they concluded that 15 genes, including GINS3, affect cardiac repolarization and that the human GINS3 ortholog is located at the $16 \mathrm{q} 21$ locus, which is strongly associated with the QT interval [14]. Furthermore, small molecules identified at the whole-organism level might be more relevant and convincing than those identified by in vitro and cell-culture-based screens.

Additional zebrafish models, such as for atherosclerosis, cardiomyopathy, arrhythmias and other cardiovascular diseases, have been established, and the related technology is already quite mature. Indeed, the zebrafish is becoming increasingly important in cardiac development and disease research.

\section{References}

1. Howe K, Clark MD, Torroja CF, Torrance J, Berthelot C, et al. (2013) The zebrafish reference genome sequence and its relationship to the human genome. Nature 496: 498-503.

2. Aidas N, Ekker SC. (2000) Effective targeted gene 'knockdown' in zebrafish. Nature Genetics 26: 216-220.
3. Suster ML, Kikuta H, Urasaki A, Asakawa K, Kawakami K (2009) Transgenesis in zebrafish with the tol2 transposon system. Methods Mol Biol. 561: 41-63.

4. Kenneth P, Wilson LG, Keating MT (2002) Heart Regeneration in Zebrafish. Science 298: 2188-2190.

5. Zhang RL, Han P, Yang H, Ouyang K, Lee D (2013) In vivo cardiac reprogramming contributes to zebrafish heart regeneration. Nature 498: 497-501.

6. Curado S (2007) Conditional targeted cell ablation in zebrafish: a new tool for regeneration studies. Dev. Dyn. 236: 1025-1035.

7. de Pater E, Clijsters L, Marques SR, Lin YF, Garavito-Aguilar ZV, et al. (2009) Distinct phases of cardiomyocyte differentiation regulate growth of the zebrafish heart. Development 136: 1633-1641.

8. Feil S, , , , , et al. (2014) Transdifferentiation of vascular smooth muscle cells to macrophage-like cells during atherogenesis. Circ Res. 115: 662-667.

9. Liu JD, . (2012) Zebrafish in the Study of Early Cardiac Development. Circ Res. 11: 870-874.

10. Zhou Y, Cashman TJ, Nevis KR, Obregon P, Carney SA, Liu Y, et al.(2011) Latent TGF- $\beta$ binding protein 3 identifies a second heart field in zebrafish. Nature 474: 645-648.

11. Daniela P, Werdich AA, Macrae CA (2010) Wnt11 patterns a myocardial electrical gradient through regulation of the L-type Ca2+ channel. Nature 466: 874-878.

12. Li J, , , , , et al. (2014) Tom70 serves as a molecular switch to determine pathological cardiac hypertrophy. Cell Res. 24: 977-993.

13. Rubinstein A L (2003) Zebrafish: from disease modeling to drug discovery. Curr. Opin. Drug Discov. Devel. 6: 218-223.

14. David M, , , , , et al. (2009) Drug-sensitized zebrafish screen identifies multiple genes, including GINS3, as regulators of myocardial repolarization. Circulation. 120: 553-539. 